\title{
Reflets
}

Revue ontaroise d'intervention sociale et communautaire

\section{Le centre national de formation en santé}

\section{Pierre Gaudet}

Volume 8, numéro 1, printemps 2002

La réadaptation : son visage français en Ontario

URI : https://id.erudit.org/iderudit/026376ar

DOI : https://doi.org/10.7202/026376ar

Aller au sommaire du numéro

Éditeur(s)

Reflets : Revue ontaroise d'intervention sociale et communautaire

ISSN

1203-4576 (imprimé)

1712-8498 (numérique)

Découvrir la revue

Citer cet article

Gaudet, P. (2002). Le centre national de formation en santé. Reflets, 8(1),

106-113. https://doi.org/10.7202/026376ar

Tous droits réservés (C) Reflets : Revue ontaroise d'intervention sociale et communautaire, 2002

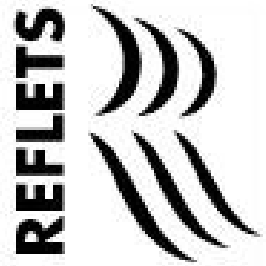

Ce document est protégé par la loi sur le droit d'auteur. L'utilisation des services d'Érudit (y compris la reproduction) est assujettie à sa politique d'utilisation que vous pouvez consulter en ligne.

https://apropos.erudit.org/fr/usagers/politique-dutilisation/ 


\title{
Le centre national de formation en santé
}

\author{
Pierre Gaudet
}

Centre national de formation en santé

\section{Quoi, qui et pourquoi?}

\section{Objectifs}

En quoi consiste le Centre national de formation en santé (CNFS) et pourquoi un tel projet ? La question se posait lors de sa création et se pose encore pour de nombreuses personnes, même parmi celles qui connaissent plus ou moins cette initiative ou en ont entendu parler.

C'est le 6 janvier 1999 que le député fédéral Mauril Bélanger annonçait, grâce à un appui financier du ministère du Patrimoine canadien de dix millions de dollars répartis sur une période de cinq années financières (1998-1999 à 2002-2003), le lancement du projet dès lors baptisé Centre national de formation en santé et dont la gestion était confiée à l'Université d'Ottawa.

L'annonce mettait en relief trois objectifs :

- Élargir, à l'Université d'Ottawa, les capacités d'accueil dans les disciplines relatives à la santé afin d'augmenter le bassin de professionnels francophones de la santé en provenance des 
communautés francophones en situation minoritaire. Cet objectif était chiffré à 90 étudiants additionnels sur cinq ans de formation, soit 30 en médecine et 60 dans les autres disciplines relatives à la santé, ces étudiants devant provenir par ailleurs des provinces autres que le Québec et l'Ontario, puisqu'on considérait que les étudiants francophones de ces deux provinces avaient déjà accès « régulièrement " à des programmes relatifs à la santé en français.

- Établir des partenariats, tout particulièrement avec l'Hôpital Montfort, pour favoriser la formation clinique en français; on se rappellera en effet que l'existence même de cette institution francophone unique en Ontario était alors remise en question dans le cadre d'une restructuration projetée du système hospitalier dans la province.

- Favoriser également le développement d'un réseau de partenariats avec les autres établissements d'enseignement post-secondaire et de santé de la francophonie canadienne pour accroître et faciliter la formation de professionnels de la santé qui oeuvreront dans les communautés francophones minoritaires.

\section{Et trois ans après?}

La mise en œuvre d'un tel projet avance par tours et détours prévus et imprévus, certains facteurs jouent beaucoup plus qu'on l'anticipait, d'autres moins, et il est certes utile et éclairant de procéder à une rétrospective au stade actuel.

\section{À l’Université d'Ottawa}

L'Université d'Ottawa avait en place 12 programmes dans 9 domaines professionnels, soit l'activité physique, l'audiologie, l'ergothérapie, la médecine, l'orthophonie, la physiothérapie, les 
sciences infirmières, le service social et le doctorat en psychologie clinique et, dans chacun, on s'est assuré de pouvoir accueillir des candidats « CNFS » additionnels.

À cet égard, disons que le premier objectif de 90 étudiants mentionné ci-haut est en bonne voie de se réaliser; il y a actuellement quelque 70 nouveaux étudiants (le chiffre fluctue, certains modifiant leur programme ou se désistent) en provenance des communautés francophones hors Ontario et hors Québec, répartis sur trois années scolaires.

La majorité de ces étudiants qui sont en sciences de la santé sont dans les disciplines de la réadaptation (audiologie/orthophonie, ergothérapie, physiothérapie), des programmes qui ne sont pas disponibles ailleurs hors-Québec; une vingtaine sont en médecine, et bien que le Programme de formation en médecine Acadie-Université de Sherbrooke accueille pour sa part une vingtaine de candidats par année et couvre principalement les besoins du Nouveau-Brunswick, on reconnaît que c'est encore insuffisant et que ça ne couvre pas les besoins des autres régions francophones, particulièrement l'Ouest. L'ajout provenant d'Ottawa devrait être bénéfique.

Les étudiants proviennent jusqu'ici de toutes les provinces canadiennes (on exclut toujours le Québec et l'Ontario pour les fins de ce projet), avec une majorité du Nouveau-Brunswick.

\section{À Montfort}

Le partenariat avec l'Hôpital Montfort est aussi en très bonne voie de réalisation. Dans un premier temps, ce partenariat s'est concentré sur l'établissement d'un centre de ressources en formation clinique accueillant principalement les étudiants de l'Université d'Ottawa, mais avec une portée beaucoup plus large, en visant à appuyer ailleurs au pays des précepteurs cliniques qui pourront accueillir en stage clinique ou pratique dans leur propre région des étudiants complétant leur formation. 
Montfort dispose maintenant d'une équipe de conseillers et conseillères en formation clinique et développement qui comprend cinq médecins couvrant diverses spécialités, un psychologue, des spécialistes en activité physique, en ergothérapie, en audiologie/ orthophonie, en physiothérapie et en sciences infirmières. L'équipe dispose aussi d'un centre multimédia qui rend disponibles des ressources de formation clinique à distance, là où les besoins existent. Il y a ainsi une consolidation du rôle de centre hospitalier universitaire de Montfort, tout en mettant à la disposition des étudiants un milieu de formation francophone privilégié.

L'équipe de conseillères en sciences de la santé (disciplines autres que la médecine) à Montfort a aussi mis sur pied une série d'ateliers de perfectionnement en français, accessibles à distance, principalement via le réseau Internet, sur L'art de la supervision clinique. Ces ateliers sont offerts depuis janvier 2002. Ils fournissent une aide précieuse aux professionnels qui ont des appréhensions à l'égard de la supervision clinique ou qui veulent enrichir leur expérience à cet égard.

\section{Ailleurs au pays}

Le troisième volet du projet, jeter les bases d'un réseau de formation fonctionnant par partenariats inter-institutionnels et inter-régionaux, progresse également, même si cette dimension est la plus complexe et la plus progressive, étant donné les nombreux intervenants et facteurs en jeu.

Résumons les développements à ce titre par des activités sur trois plans :

- celui très concret de l'enseignement et de la formation clinique comme tels;

- la liaison et des échanges inter-institutionnels visant la planification de champs d'intervention spécifiques;

- la représentation des dirigeants des institutions et des réseaux principaux, pour traiter des orientations et de la gouvernance d'ensemble. 


\section{Voyons donc plus en détails chacun de ces plans}

L'enseignement et la formation clinique résultant de partenariats comprennent un nouveau programme de baccalauréat en sciences infirmières au Collège universitaire de Saint-Boniface (CUSB), avec une vingtaine d'étudiants en formation dans la première année qui est en cours depuis septembre 2001. Les collaborations à ce projet incluent, en plus de financements à la fois fédéral et provincial, et la collaboration du CUSB et du CNFS, celle du département de Sciences infirmières de l'Université d'Ottawa et celle des milieux où la formation clinique peut avoir lieu,jusqu'à maintenant, l'Hôpital général de Saint-Boniface, le Centre de Santé Saint-Boniface, le Centre Taché (soins de longue durée) et des garderies.

Un nouveau programme de sciences infirmières est aussi en élaboration à la Faculté Saint-Jean d'Edmonton et vise un lancement en septembre 2003. Les autres programmes sont :

- un programme post-diplôme en sciences infirmières, offert à distance par la collaboration entre l'Université Laurentienne, l'Université d'Ottawa, l'Hôpital Montfort;

- une série de cours en périnatalogie offerts à distance et donnés en collaboration par l'Hôpital Montfort et l'Université d'Ottawa;

- le programme d'infirmière praticienne coordonné par l'Université d'Ottawa est donné à distance au Manitoba avec des infirmières sur place comme tutrices;

- les coordonnateurs de stages cliniques à l'Université d'Ottawa dans les disciplines de la réadaptation (audiologie/orthophonie, ergothérapie, physiothérapie) favorisent et cherchent activement des stages dans les régions d'où les étudiants proviennent.

Mentionnons enfin la planification en cours d'un baccalauréat en sciences de la santé qui pourrait s'offrir en partenariat interinstitutionnel, de même qu'un certificat en gérontologie.

On voit que c'est surtout en sciences infirmières que les principales activités se sont réalisées jusqu'ici; notons qu'on pouvait 
s'appuyer sur une expérience à l'échelle ontarienne d'échanges inter-institutionnels dans ce domaine, qu'on y connaît aussi une pénurie partout invoquée.

Ces partenariats ont donc permis d'inscrire une quarantaine d'étudiants à travers le pays, ce qui a pu se faire grâce aux technologies d'enseignement à distance, permettant de surmonter les facteurs d'éloignement et de dispersion — qui sont prédominants dans les communautés minoritaires. Ce sont par conséquent des expériences prometteuses qui ajoutent un contingent important d'étudiants à celui qui oeuvre sur place à Ottawa.

Les partenariats ont pris forme par la mise sur pied de cinq Groupes de travail dans des domaines déterminants de la formation dans le champ de la santé : la programmation, la formation clinique, le recrutement, la médecine, la médiatisation (ou la formation à distance). Ces groupes réunissent une soixantaine de membres venant des diverses institutions d'enseignement postsecondaires et des diverses régions du pays. Il s'agit de personnes directement impliquées dans les champs d'activités visées par les Groupes de travail.

Chaque Groupe se réunit régulièrement par conférence téléphonique et, en personnes, au moins une fois par année. Des plans de travail initiaux dans le domaine de chaque Groupe ont été élaborés et servent déjà de jalons concrets pour construire le réseau d'avenir (dont nous parlerons plus loin). On ne peut tout recenser. Mentionnons cependant que c'est sous l'impulsion de ces Groupes, particulièrement celui sur le recrutement, que l'on a mis en œuvre diverses activités de promotion du CNFS et d'appui aux étudiants.

L'esprit de partenariat s'est actualisé par la création en novembre 2000 d'un Conseil national d'orientation du CNFS; il réunit une trentaine de personnes, des dirigeants des diverses institutions et des représentants des diverses régions et des secteurs aptes à jouer un rôle direct ou d'appui dans le domaine de la formation de professionnels en santé. Il est présidé par Mme Huguette Labelle. Le Conseil a donné lieu à des développements sur deux plans principaux : 
- L'élaboration du concept d'un Consortium pan-canadien de formation de professionnels francophones de la santé, qui fonctionnera sur une base inter-institutionnelle et interrégionale et qui prendrait sa pleine expression dans une deuxième phase (avril 2003 à avril 2008), laquelle doit venir consolider et élargir les assises établies par la première phase du Centre national de formation en santé dont le financement échoit à la fin mars 2003.

- Des mesures en vue de favoriser le financement requis de cette deuxième phase.

En ce sens, le Conseil a permis la liaison et la collaboration avec le Comité consultatif des communautés francophones en situation minoritaire nommé au printemps 2000 par le Ministre fédéral de la Santé pour le conseiller relativement à l'accès des communautés francophones minoritaires à des services de santé dans leur langue. Ce Comité a favorisé par l'entremise de la Fédération des communautés francophones et acadienne du Canada (FCFA) une étude majeure Santé en français et a déposé un Rapport au Ministre fédéral de la Santé en septembre 2001. Dans un cadre d'ensemble visant à mettre en place une politique globale de la santé dans les communautés francophones minoritaires, ce rapport souligne dans ses recommandations l'importance de la formation et, en particulier, la nécessité de financer une deuxième phase sous l'égide d'un Consortium tel que celui mentionné ci-haut.

Cette collaboration a donné lieu, en novembre 2001, à une présentation des recteurs de l'Université d'Ottawa et de l'Université de Moncton, MM. Gilles Patry etYvon Fontaine, sur la problématique d'avenir de la formation dans le domaine de la santé lors du Forum "Santé en français " organisé le Comité consultatif à Moncton du 2 au 4 novembre 2001.

\section{Et l'avenir?}

Comme l'indiquait M. Gilles Patry : «...on est à établir des bases pour l'avenir et nous sommes heureux qu'elles répondent aux 
objectifs fixés pour le projet CNFS et qu'elles puissent servir à édifier un avenir solide et encore meilleur grâce à des partenariats concrets. Déjà ils se révèlent réalisables, il faut les élargir et les multiplier".

L'évolution, et les travaux qu'elle a générés et qu'on vient d'esquisser, permettent en effet de dégager trois constatations principales :

- les besoins des communautés sont très grands : on estime qu'il faudrait tripler, voir quadrupler le nombre de professionnels francophones pour y donner de pleins services;

- des capacités de formation existent et offrent une certaine assise; mais on doit l'élargir et prodiguer une formation qui se fasse autant que possible dans le milieu de provenance des candidats ou en lien avec celui-ci;

- on ne pourra pas, avec les nombres en cause, multiplier programmes et infrastructures, mais on pourra souvent offrir ces programmes, en tout ou en partie, en partenariat et à distance, selon diverses modalités. Des exemples concrets récents le démontrent.

Pour terminer cette recension sommaire de l'initiative de formation en cours (faute de place on a pu mentionner de nombreuses collaborations et projets), disons qu'elle s'inscrit dans une problématique beaucoup plus large de développement des communautés francophones, mais qu'elle y joue un rôle déterminant. Elle a déjà donné des fruits mais a surtout jeté les fondements de ce qui peut devenir une réalisation majeure : équiper en ressources humaines suffisantes la prochaine génération dans un domaine qui implique directement son bien-être. 